\title{
A formula for calculating the rotational period of planet or star
}

xiaozhong zhai ( $\nabla$ zxzdcoyk@yahoo.com )

odense kommune https://orcid.org/0000-0002-0004-4260

\section{Research Article}

Keywords: acceleration, gravity, mass, period, rotation

Posted Date: September 7th, 2021

DOI: https://doi.org/10.21203/rs.3.rs-859954/v2

License: (9) This work is licensed under a Creative Commons Attribution 4.0 International License. Read Full License 


\title{
A formula for calculating the rotational period of planet or star
}

\author{
Author:Xiaozhong Zhai
}

Affiliation: Odense kommune In Denmark

Abstract: This paper reports a formula for calculating the rotation period of planet or star. The rotational radius, mass and gravitational acceleration on its surface, the three factors, determine the rotational period of planet or star.

Key word: acceleration, gravity, mass, period, rotation.

\section{1 , Introduction}

Thus far, a unique formula for calculating the rotation period of planet in the solar system and star, for example, the Sun, remains a challenge. This puzzles astronomer for centuries. The next section represents my attempt to derive such a formula from the traditional theory of physics.

\section{Theory and formula}

Suppose a planet or star is a sphere with a uniform distribution of matter. If we let its rotational speed in the tangential direction be equal to its orbit velocity, its radius would expand by $\mathrm{X}$ times. Then, we have the following equation.

$$
2 \pi r X=t v
$$

The value of $x$ may be calculated by the following equation:

$$
X^{2}=\frac{v^{2} r}{G m-g r^{2}}
$$


So, $t$, the rotation period of a planet or star may be calculated by the following formula:

$$
t^{2}=\frac{4 \pi^{2} r^{3}}{G m-g r^{2}}
$$

The $g$ is the gravity on the surface of a planet or star. $G$ is the gravitational constant. $m$ is its mass. $t$ represents its rotation period. $r$ is its radius and $v$ its orbit average velocity.

\section{Discussion}

In the above equation or formula, the gravity (g) represents the difference $\left(\frac{G m}{r^{2}}-\frac{v^{2}}{r x^{2}}\right)$ between centripetal acceleration coming from mass or mass distribution $\left(\frac{G m}{r^{2}}\right)$ and the centrifugal acceleration $\left(\frac{v^{2}}{r x^{2}}\right)$ coming from the rotation, called net centripetal acceleration (Richard.B.2007, Hofmann-Wellenhof.B.etal.2006). In the process of rotation, two opposite effects form a pair to impart to the rotation object at the same times.

The value for $g, \frac{G m}{r^{2}}$ and $\frac{v^{2}}{r x^{2}}$ of planet in solar system and of Sun are shown in table 1 and that for rotation period $t$ and $x$ in table 2 .

The gravity (g) on the Earth's surface varies around from $9.7639 \mathrm{~m} / \mathrm{s}^{2}$ on the Nevado Huascarán mountain in Peru to $9.8337 \mathrm{~m} / \mathrm{s}^{2}$ at the surface of the Arctic Ocean(Christian.H.et al.2013) because the earth is a sphere with uneven distribution of matter. In 1901 the third General Conference on weights and Measures defined a standard gravitational acceleration ( $g$ ) for the surface of the Earth : $g=9.80665$.

The average gravitational acceleration or the net gravity (g) at the surface of the Earth is $9.78638037 \frac{\mathrm{m}}{\mathrm{s}^{2}}$ (Qotze.H.2014)with even distribution of matter in table 1 .

The rotation period ( $t$ ) of planet calculated by the equations is 100 percent consistent with the observations in the literature (Helled.R.et al.2015, Kutna.M.L.2003, NASA,Wikipedia) in a accurate to seconds in table 2 .

\section{4, Conclusion}


There are the three factors that determine the rotational period of planet or star. They are rotational radius (r), mass and gravitational acceleration ( $g$ ) on the surface of planet and star.

\section{Reference:}

Christian. H. et al. 2013, New Ultrahigh-Resolution Picture of Earth's gravity field. Geophysical Research Letters. 40(16):4279-4283.

Helled. R., Galanti. E. and Kaspi. Y. 2015, Nature. 2015. 520, 202204 .

Hofmann-Wellenhof. B., Moritz. H. 2006, Physical Geodesy (2 nd Ed). Springer. ISBN 978-3-211-33544-4. \$ 2.1 .

Kutna. M.L. 2003, system, Physical perspective, 2end Cambridge university

NASA planetary fact sheet and solar system by Wikipedia in internet.

Qotze.H. 2014, International Gravity Formula.In:Gupta H.K. Encyclopedia of Solid Earth Geophysics.Encyclopedia of Earth Science Series. Springer Dordrecht.

Richard. B. 2007, Precise Measurement of Mass (PDF). Sawe Paper No.3147. Arlington, Texas: S.A.W.E., INC. Retrieved.

Table 1: The value of the centripetal gravitational acceleration $\left(\frac{G m}{r^{2}}\right)$, of the centrifugal gravitational acceleration $\left(\frac{v^{2}}{r X^{2}}\right)$ and of the gravitational acceleration $(g)$ on the surface of object $\left(\frac{m}{s^{2}}\right)$

\begin{tabular}{|c|c|c|c|c|c|c|c|c|c|c|c|c|}
\hline & $\begin{array}{l}\text { Mercu } \\
\text { ry }\end{array}$ & Venus & Earth & Mars & $\begin{array}{l}\text { Cere } \\
\mathrm{s}\end{array}$ & $\begin{array}{l}\text { Jupit } \\
\text { er }\end{array}$ & $\begin{array}{l}\text { Satur } \\
\mathrm{n}\end{array}$ & $\begin{array}{l}\text { Halle } \\
y\end{array}$ & $\begin{array}{l}\text { Uran } \\
\text { ia }\end{array}$ & $\begin{array}{l}\text { Neptu } \\
\text { ne }\end{array}$ & Pluto & Sun \\
\hline $\mathrm{Gm}$ & 3.701 & 8.870 & 9.820 & 3.72 & 0.28 & 25.92 & 11.18 & 1.213 & 9.00 & 11.27 & 0.617 & 273.6 \\
\hline$\overline{r^{2}}$ & 49559 & 09425 & 25812 & $\begin{array}{l}7854 \\
37\end{array}$ & $\begin{array}{l}0203 \\
43\end{array}$ & 03022 & $\begin{array}{l}60361 \\
4\end{array}$ & $\begin{array}{r}46909 \\
\times 10^{-4}\end{array}$ & $\begin{array}{l}7601 \\
74\end{array}$ & $\begin{array}{l}45662 \\
4\end{array}$ & 21204 & $\begin{array}{l}98125 \\
09\end{array}$ \\
\hline$\frac{v^{2}}{r X^{2}}$ & $\begin{array}{r}3.751 \\
26284 \\
\times 10^{-6}\end{array}$ & $\begin{array}{r}5.418 \\
15969 \\
\times 10^{-7}\end{array}$ & $\begin{array}{l}0.033 \\
87777 \\
6\end{array}$ & $\begin{array}{l}0.01 \\
7028 \\
65\end{array}$ & $\begin{array}{l}0.01 \\
7495 \\
93\end{array}$ & $\begin{array}{l}2.162 \\
62771\end{array}$ & $\begin{array}{l}1.593 \\
44308\end{array}$ & $\begin{array}{l}1.202 \\
15717 \\
\times 10^{-5}\end{array}$ & $\begin{array}{l}0.25 \\
9947 \\
2\end{array}$ & $\begin{array}{l}0.289 \\
39436\end{array}$ & $\begin{array}{r}1.538 \\
66659 \\
\times 10^{-4}\end{array}$ & $\begin{array}{l}0.005 \\
86868\end{array}$ \\
\hline $\mathrm{g}$ & $\begin{array}{l}3.701 \\
49184\end{array}$ & $\begin{array}{l}8.870 \\
09371\end{array}$ & $\begin{array}{l}9.786 \\
38034 \\
4\end{array}$ & $\begin{array}{l}3.71 \\
0825 \\
72\end{array}$ & $\begin{array}{l}0.26 \\
2707 \\
5\end{array}$ & $\begin{array}{l}23.75 \\
76744 \\
9\end{array}$ & $\begin{array}{l}9.592 \\
59306\end{array}$ & $\begin{array}{l}0.000 \\
10932 \\
5\end{array}$ & $\begin{array}{l}8.74 \\
7654 \\
54\end{array}$ & $\begin{array}{l}10.98 \\
51718 \\
8\end{array}$ & $\begin{array}{l}0.617 \\
05817\end{array}$ & $\begin{array}{l}273.6 \\
92256 \\
41\end{array}$ \\
\hline
\end{tabular}


Note: the unit of $g$ is $\frac{m}{s^{2}}$.

Table 2 : The $\mathrm{X}$ value calculated by formula, the rotation period ( $t$ ) in second calculated by formula, the rotation period in second by observations $\left(t_{b}\right)$ and the accuracy rate between the calculating and the observed value.

\begin{tabular}{|c|c|c|c|c|c|c|c|c|c|c|c|c|}
\hline & $\begin{array}{c}\text { Mercu } \\
\text { ry }\end{array}$ & Venus & $\begin{array}{c}\text { Eart } \\
h\end{array}$ & Mars & $\begin{array}{c}\text { Cere } \\
\text { s }\end{array}$ & $\begin{array}{l}\text { Jupi } \\
\text { ter }\end{array}$ & $\begin{array}{c}\text { Satur } \\
\mathrm{n}\end{array}$ & Halley & $\begin{array}{c}\text { Urani } \\
\text { a }\end{array}$ & $\begin{array}{c}\text { Nept } \\
\text { une }\end{array}$ & Pluto & Sun \\
\hline$x$ & $\begin{array}{l}15824 \\
.4389 \\
467\end{array}$ & $\begin{array}{l}19,34 \\
0.185 \\
37754\end{array}$ & $\begin{array}{l}64.1 \\
1126 \\
23\end{array}$ & $\begin{array}{l}100.4 \\
36372 \\
25\end{array}$ & $\begin{array}{l}196 . \\
8131 \\
1443\end{array}$ & $\begin{array}{l}1.06 \\
1808 \\
09\end{array}$ & $\begin{array}{l}0.998 \\
86312\end{array}$ & $\begin{array}{l}19,395 \\
.19834 \\
975\end{array}$ & $\begin{array}{l}2.647 \\
26349 \\
2\end{array}$ & $\begin{array}{c}2.03 \\
5560 \\
73\end{array}$ & $\begin{array}{l}350.75 \\
202619\end{array}$ & $\begin{array}{c}108.82 \\
816\end{array}$ \\
\hline$t$ & $\begin{array}{c}50679 \\
75.86 \\
51213 \\
3\end{array}$ & $\begin{array}{c}21129 \\
193.4 \\
88314 \\
37\end{array}$ & $\begin{array}{l}8616 \\
4.10 \\
0352\end{array}$ & $\begin{array}{c}88645 \\
.6804 \\
1193\end{array}$ & $\begin{array}{c}3266 \\
9.45 \\
7480 \\
48\end{array}$ & $\begin{array}{c}3572 \\
4.13 \\
6804 \\
87\end{array}$ & $\begin{array}{c}37983 \\
.2781 \\
9036\end{array}$ & $\begin{array}{c}190062 \\
.12631 \\
611\end{array}$ & $\begin{array}{l}62062 \\
.4685 \\
198\end{array}$ & $\begin{array}{l}5795 \\
5.74 \\
5999 \\
66\end{array}$ & $\begin{array}{c}551856 \\
.672\end{array}$ & $\begin{array}{l}216431 \\
7.9658 \\
340\end{array}$ \\
\hline$t_{b}$ & $\begin{array}{l}50679 \\
75,91 \\
7956\end{array}$ & $\begin{array}{l}21129 \\
192,1 \\
79934\end{array}$ & $\begin{array}{l}8616 \\
4,07 \\
1519 \\
54\end{array}$ & $\begin{array}{l}88645 \\
, 6675 \\
26916\end{array}$ & $\begin{array}{l}3266 \\
9,45 \\
6439 \\
186\end{array}$ & $\begin{array}{l}3572 \\
4,13 \\
6766 \\
504\end{array}$ & $\begin{array}{l}37983 \\
, 2781 \\
36837\end{array}$ & $\begin{array}{l}190059 \\
, 46007 \\
248\end{array}$ & $\begin{array}{l}62062 \\
, 4686 \\
6021\end{array}$ & $\begin{array}{l}5795 \\
5,74 \\
6459 \\
929\end{array}$ & $\begin{array}{l}551867 \\
, 79218 \\
384\end{array}$ & $\begin{array}{l}2164 \\
317,63 \\
53269\end{array}$ \\
\hline * & $100 \%$ & $100 \%$ & $100 \%$ & $100 \%$ & $100 \%$ & $100 \%$ & $100 \%$ & $100 \%$ & $100 \%$ & $100 \%$ & $100 \%$ & $100 \%$ \\
\hline
\end{tabular}

Note: $* *$ the accuracy rate rate $\left(\frac{t}{t_{b}} 100 \%\right)$

Table 3: the value of mass $(\mathrm{kg})$, average radius $(\mathrm{m})$ and orbital velocity $\left(\frac{\mathrm{m}}{\mathrm{s}^{2}}\right)$ by observation.

\begin{tabular}{|c|c|c|c|c|c|c|c|c|c|c|c|c|}
\hline & $\begin{array}{l}\text { Mercu } \\
\text { ry }\end{array}$ & Venus & $\begin{array}{l}\text { Eart } \\
\text { h }\end{array}$ & Mars & Ceres & $\begin{array}{l}\text { Jupi } \\
\text { ter }\end{array}$ & $\begin{array}{l}\text { Satu } \\
\text { rn }\end{array}$ & $\begin{array}{l}\text { Hall } \\
\text { ey }\end{array}$ & $\begin{array}{l}\text { Uran } \\
\text { ia }\end{array}$ & $\begin{array}{l}\text { Neptu } \\
\text { ne }\end{array}$ & Pluto & Sun \\
\hline$r$ & $\begin{array}{l}2.43 \\
97 \times \\
10^{6}\end{array}$ & $\begin{array}{l}.05 \\
18 \times \\
10^{6}\end{array}$ & $\begin{array}{l}6.3 \\
71 \times \\
10^{6}\end{array}$ & $\begin{array}{l}3.38 \\
95 \times \\
10^{6}\end{array}$ & $\begin{array}{r}4.73 \\
\times 10^{5}\end{array}$ & $\begin{array}{l}6.99 \\
11 \times \\
10^{7}\end{array}$ & $\begin{array}{l}5.82 \\
32 \times \\
10^{7}\end{array}$ & $\begin{array}{l}1.1 \times \\
10^{4}\end{array}$ & $\begin{array}{l}2.53 \\
62 \times \\
10^{7}\end{array}$ & $\begin{array}{l}2.46 \\
22 \times \\
10^{7}\end{array}$ & $\begin{array}{l}1.18 \\
7 \times \\
10^{6}\end{array}$ & $\begin{array}{l}6.963 \\
4 \times 10^{8}\end{array}$ \\
\hline $\mathrm{v}$ & $\begin{array}{l}4787 \\
2.40 \\
8916 \\
53\end{array}$ & $\begin{array}{l}3502 \\
1.02 \\
2076 \\
51\end{array}$ & $\begin{array}{l}297 \\
84 . \\
860 \\
790 \\
3\end{array}$ & $\begin{array}{l}1790 \\
4.12 \\
4374 \\
61\end{array}$ & $\begin{array}{l}1790 \\
4.12 \\
4374 \\
61\end{array}$ & $\begin{array}{l}1305 \\
5.98 \\
5798 \\
55\end{array}$ & $\begin{array}{l}9621 \\
.773 \\
0449 \\
8\end{array}$ & $\begin{array}{l}7052 \\
.956 \\
3108 \\
7\end{array}$ & $\begin{array}{l}6797 \\
.222 \\
8212 \\
4\end{array}$ & $\begin{array}{l}5433 \\
.638 \\
672\end{array}$ & $\begin{array}{l}4740 \\
.212 \\
4793 \\
3\end{array}$ & $\begin{array}{l}22000 \\
0\end{array}$ \\
\hline $\mathrm{m}$ & $\begin{array}{l}3.3011 \\
\times \\
10^{23}\end{array}$ & $\begin{array}{l}4.8675 \\
\times \\
10^{24}\end{array}$ & $\begin{array}{l}5.972 \\
37 \times \\
10^{24}\end{array}$ & $\begin{array}{l}6.4171 \\
\times \\
10^{23}\end{array}$ & $\begin{array}{l}9.393 \\
\times \\
10^{20}\end{array}$ & $\begin{array}{c}1.898 \\
19 \times \\
10^{27}\end{array}$ & $\begin{array}{l}5.683 \\
\times \\
10^{26}\end{array}$ & $\begin{array}{l}2.2 \times \\
10^{14}\end{array}$ & $\begin{array}{c}8.681 \\
\times \\
10^{25}\end{array}$ & $\begin{array}{c}1.0241 \\
\times \\
10^{26}\end{array}$ & $\begin{array}{c}1.303 \\
\times \\
10^{22}\end{array}$ & $\begin{array}{r}1.9885 \\
\times 10^{30}\end{array}$ \\
\hline
\end{tabular}

Note: r represents radius, $\mathrm{v}$ is average orbital velocity and $\mathrm{m}$ mass. 